Supporting Information for

\title{
A Homochiral Nanotubular Crystalline Framework of Metallomacrocycles for Enantioselective Recognition and Separation
}

\author{
Gao Li, Weibin Yu and Yong Cui* \\ School of Chemistry and Chemical Technology, Shanghai Jiao Tong University, \\ Shanghai 200240, China. \\ Email: yongcui@sjtu.edu.cn
}

\section{Table of Content}

1. Materials and General Procedures

2. Synthesis

3. Guest-exchange experiment

4. Separation and recycling experiments

5. Table S1. Crystal data and structure refinement for $\mathbf{1} \cdot 6 \mathrm{THF}$

6. Table S2. Selected bond lengths and angles for $\mathbf{1} \cdot 6 \mathrm{THF}$

7. Table S3. Crystal data and structure refinement for 1·6(2-pentanol)

8. Table S4. Selected bond lengths and angles for 1·6(2-pentanol)

9. Figure S1. The molecular structure of $1 \cdot 6 \mathrm{THF}$.

10. Figure S2. The molecular structure of $\mathbf{1} \cdot 6$ pentanol

11. Figure S3. $\mathrm{CH} \cdots \pi$ interactions between the butyl groups and the coordinated pyridine rings of $\mathbf{L}$ direct $\mathrm{Zn}_{6} \mathrm{~L}_{6}$ macrocycles assembling into nanotubular structure.

12. Figure S4. Intermolecular $\pi-\pi$ interaction between $\mathrm{Zn}_{6} \mathrm{~L}_{6}$ macrocycles.

13. Figure S5. $\mathrm{CH} \cdots \pi$ interactions between uncoordinated pyridyl groups and aromatic rings of $\mathrm{Zn}_{6} \mathrm{~L}_{6}$ macrocycles.

14. Figure S6. $\mathrm{CH} \cdots \mathrm{N}$ interactions between uncoordinated pyridyl groups and aromatic rings of $\mathrm{Zn}_{6} \mathrm{~L}_{6}$ macrocycles.

15. Figure S7. A view of the packing of $\mathrm{Zn}_{6} \mathrm{~L}_{6}$ cages.

16. Figure S8. The PXRD patterns of 1 and its adducts.

17. Figure S9. The PXRD patterns of 1 after the $3^{\text {rd }}$ and $5^{\text {th }}$ cycles of resolution.

18. Figure S10. TGA curve of $\mathbf{1}$ and its adducts

19. Figure S11. The $\mathrm{N}_{2}$ adsorption isotherm of $\mathbf{1}$.

20. Figure S12.Chiral GC results of the desorbed (R)-3-methyl-2-butanol and (R)-2-pentanol from (R)-1.

21. Figure S13. Chiral GC results of recycling and reuse of (R)-1 for separation of racemic 2-butanol

22. Figure S14. Chiral GC of the desorbed (S)-2-Butanol, (S)-3-methyl-2-butanol and (S)-2-pentanol from (S)-1.

23. Figure S15. CD spectra of (R)- and (S)-1. 


\section{Materials and General Procedures.}

All of the chemicals are commercial available, and used without further purification. Elemental analyses of $\mathrm{C}, \mathrm{H}$ and $\mathrm{N}$ were performed with an EA1110 CHNS-0 CE elemental analyzer. The IR (KBr pellet) spectrum was recorded (400-4000 $\mathrm{cm}^{-1}$ region) on a Nicolet Magna 750 FT-IR spectrometer. The solid state CD spectra were recorded on a J-800 spectropolarimeter (Jasco, Japan).. Thermogravimetric analyses (TGA) were carried out in an air atmosphere with a heating rate of $10 \mathrm{C} \mathrm{min}^{-1}$ on a STA449C integration thermal analyzer. Powder X-ray diffraction (PXRD) data were collected on a DMAX2500 diffractometer using $\mathrm{Cu} \mathrm{K} \alpha$ radiation. The calculated PXRD patterns were produced using the SHELXTL-XPOW program and single crystal reflection data. Gas chromatography was conducted on Lunan Ruihong SP-6890 equipped with a flame ionization detector. The $\mathrm{N}_{2}$ adsorption isotherms was measured at $77 \mathrm{~K}$ by using a Micromeritics ASAP $2010 \mathrm{M}+\mathrm{C}$ system. Before the adsorption measurement, the sample was outgassed at $398 \mathrm{~K}$ in the port of the adsorption analyzer for $2 \mathrm{~h}$. All the surface areas were calculated based on multi-point BET plots, while the pore volumes were estimated based on BJH method.

X-ray Crystallography. Single-crystal XRD data for the compounds was collected on on a Bruker Smart 1000 CCD diffractometer with Mo-K $\alpha$ radiation $(\lambda=0.71073 \AA)$ at room temperature. The empirical absorption correction was applied by using the SADABS program (G. M. Sheldrick, SADABS, program for empirical absorption correction of area detector data; University of Göttingen, Göttingen, Germany, 1996). The structure was solved using direct method, and refined by full-matrix least-squares on F2 (G. M. Sheldrick, SHELXTL97, program for crystal structure refinement, University of Göttingen, Germany, 1997). In both 1.6THF and 1·6(2-pentanol), the guest molecules and H-atoms were refined isotropically, while all other atoms were refined anisotropically. Crystal data and details of the data collection are given in Tables S1 and S3 while the selected bond distances and angles are presented in Tables S2 and S4. 


\section{Synthesis of 1.6THF and the apohost 1}

A mixture of $\mathrm{Zn}\left(\mathrm{NO}_{3}\right)_{2} \cdot 6 \mathrm{H}_{2} \mathrm{O}$ (14.9 mg, $\left.0.05 \mathrm{mmol}\right), \mathrm{H}_{2} \mathbf{L}(29.5 \mathrm{mg}, 0.05 \mathrm{mmol})$, DMF $(5 \mathrm{~mL})$, THF $(5 \mathrm{~mL})$ and water $(0.5 \mathrm{~mL})$ was sealed in a $10 \mathrm{~mL}$ vial with a screw cap and heated at $80{ }^{\circ} \mathrm{C}$ for 1 day. The mixture was then cooled to room temperature and light-yellow rod-like crystals were collected, washed with ether and dried in air. Yield: $28.3 \mathrm{mg}, 82 \%$ based on $\mathrm{Zn}$. Anal (\%). Calcd for $\mathrm{C}_{252} \mathrm{H}_{300} \mathrm{~N}_{24} \mathrm{O}_{18} \mathrm{Zn}_{6}$ : C, 69.65; H, 6.96; N, 7.74. Found: C, 68.98; H, 6.91; N, 7.67. IR (KBr, cm $\left.{ }^{-1}\right): 3414$ (m), 2934 (m), 1632 (s), $1586(\mathrm{~s}), 1548(\mathrm{~m}), 1526(\mathrm{~m}), 1448(\mathrm{~m}), 1434(\mathrm{~s}), 1389 \mathrm{~s}), 1384(\mathrm{~m}), 1352(\mathrm{~m}), 1290(\mathrm{~m})$, 1222(m), 1158(e), 1016(e), $814(\mathrm{w}), 642(\mathrm{w})$. The guest molecules can be readily removed to generate 1 just by evacuating $1 \cdot 6 \mathrm{THF}$ at $40^{\circ} \mathrm{C}$ for 2 hours, as shown by TGA (Figure S8) and powder XRD (Figure S9).

\section{Guest-Exchange Experiment}

A typical guest-exchange experiment: Freshly evaculated crystals of $\mathbf{1}$ $(0.10 \mathrm{mmol}, 39.1 \mathrm{mg})$ and neat 2-pentanol $(5 \mathrm{~mL})$ or a mixture of 2-pentanol (2 $\mathrm{mL})$ and $\mathrm{CH}_{2} \mathrm{Cl}_{2}(3 \mathrm{~mL})$ were placed in a $10 \mathrm{~mL}$ capped vial at $40{ }^{\circ} \mathrm{C}$ for two days. The resulting sample was then isolated by filtration, washed several times with ether. Based on the results of elemental analysis and TGA, the product can be formulated as 1.6pentanol. Powder XRD diffraction experiments indicate that crystals of $\mathbf{1}$ remained almost intact without loss of crystallininity. Similarly, the guest-exchanged products can be formulated as 1.6guests for 2-butanol and 3-methyl-2-butanol. The formulation was further confirmed by single crystal X-ray crystallography for 2-pentanol-exchanged product. Single-crystals suitable X-ray diffraction was obtained by heating crystalline sample of 1.6THF in 2-pentanol in a sealed vial at $40{ }^{\circ} \mathrm{C}$ for 3 days.

Elemental Analysis data of 1.6(2-Butanol):

Anal (\%). Calcd for C252H312N24O18Zn6: C, 69.46; H, 7.22; N, 7.71. Found: C, 70.00; H, 7.08; N, 7.70.

Elemental Analysis data of 1.6(3-methyl-2-Butanol):

Anal (\%). Calcd for C258H324N24O18Zn6: C, 69.76; H, 7.35; N, 7.57. Found: C, 69.12; H, 7.43; N, 7.44.

Elemental Analysis data of 1.6(2-pentanol):

Anal (\%). Calcd for $\mathrm{C}_{258} \mathrm{H}_{324} \mathrm{~N}_{24} \mathrm{O}_{18} \mathrm{Zn}_{6}$ : C, 69.76; H, 7.35; N, 7.57. Found: C, $68.77 ; \mathrm{H}, 7.37$; N, 7.48 . 


\section{Separation and recycling experiments}

Evacuated sample of 1 (50 $\mathrm{mg})$ and racemic 2-butanol $(5 \mathrm{~mL})$, 3-methyl-2-butanol $(5 \mathrm{~mL})$ or 2-pentanol $(5 \mathrm{~mL})$ was put in a sealed vial at 40 ${ }^{\circ} \mathrm{C}$ for two days. The solid samples were filtered, washed with diethyl ether, and then loaded into a distillation setup and gently heated to under vacuum while the volatiles were collected with a liquid nitrogen bath. The recycling and resued experiment with racemic 2-butanol were done in the same way.

Enantiomeric excess of the 2-butanol was determined to be $98.4 \%$ by using gas chromatography (GC). Conditions Column: CHIRALDEX G-TA by ASTEC Company ( $30 \mathrm{~m} \times 0.25 \mathrm{~mm}$ I.D.). Column temperature: $26^{\circ} \mathrm{C}$. Carrier gas: $\mathrm{N}_{2} 0.5 \mathrm{ml} / \mathrm{min}, \mathrm{H}_{2} 0.5 \mathrm{ml} / \mathrm{min}$. Retention time: minor compound (S), $24.67 \mathrm{~min}$., and major compound (R), $25.58 \mathrm{~min}$.

Enantiomeric excess of the desorbed 3-methyl-2-butanol was determined to be $99.6 \%$ by using GC. Conditions Column: CHIRALDEX G-TA by ASTEC Company ( $30 \mathrm{~m} \times 0.25 \mathrm{~mm}$ I.D.). Column temperature: $30^{\circ} \mathrm{C}$. Carrier gas: $\mathrm{N}_{2} 1.0 \mathrm{ml} / \mathrm{min}, \mathrm{H}_{2} 1.0 \mathrm{ml} / \mathrm{min}$. Retention time: minor compound (S), $22.22 \mathrm{~min}$., and major compound (R), $23.15 \mathrm{~min}$.

Enantiomeric excess of the desorbed 2-pentanol was determined to be 99.5\% by using GC. Conditions Column: CHIRALDEX G-TA by ASTEC Company (30 m $\times 0.25 \mathrm{~mm}$ I.D.). Column temperature: $40^{\circ}$ C. Carrier gas: $\mathrm{N}_{2}$ $1.0 \mathrm{ml} / \mathrm{min}, \mathrm{H}_{2} 1.0 \mathrm{ml} / \mathrm{min}$. Retention time: minor compound (S), $22.13 \mathrm{~min}$, and major compound (R), $22.80 \mathrm{~min}$. 
Table S1. Crystal data and structure refinement for 1·6THF.

\begin{tabular}{|c|c|}
\hline Identification code & $\mathbf{1} \cdot 6 \mathrm{THF}$ \\
\hline Empirical formula & $\mathrm{C}_{252} \mathrm{H}_{300} \mathrm{~N}_{24} \mathrm{O}_{18} \mathrm{Zn}_{6}$ \\
\hline Formula weight & 4345.38 \\
\hline Temperature & 293(2) K \\
\hline Wavelength & $0.71073 \AA$ \\
\hline Crystal system & Trigonal \\
\hline Space group & $\mathrm{R} 3$ \\
\hline \multicolumn{2}{|l|}{ Unit cell dimensions } \\
\hline & $\mathrm{a}=44.861(6) \quad \AA \quad$ alpha $=90^{\circ}$ \\
\hline & $\mathrm{b}=44.861(6) \quad \AA \quad$ beta $=90^{\circ}$ \\
\hline & $\mathrm{c}=10.203(2) \AA$ gamma $=120^{\circ}$ \\
\hline Volume, Z & $17783(5) \quad \AA^{3}, \quad 3$ \\
\hline Density (calculated) & $1.217 \mathrm{Mg} / \mathrm{m}^{3}$ \\
\hline Absorption coefficient & $0.663 \mathrm{~mm}^{-1}$ \\
\hline $\mathrm{F}(000)$ & 6912 \\
\hline Crystal size & $0.12 \times 0.06 \times 0.06 \mathrm{~mm}$ \\
\hline Theta range for data collection & 3.03 to $24.99^{\circ}$ \\
\hline Limiting indices & $-53<\mathrm{h}<53,-50<\mathrm{k}<53,-12<1<12$ \\
\hline Reflections collected & 44647 \\
\hline Independent reflections & $13876($ Rint $=0.0282)$ \\
\hline Completeness to theta & $24.99 \% 99.8 \%$ \\
\hline Refinement method & Full-matrix least-squares on $\mathrm{F}^{2}$ \\
\hline Data / restraints / parameters & $13876 / 8 / 851$ \\
\hline Goodness-of-fit on $\mathrm{F}^{2}$ & 1.065 \\
\hline Final $\mathrm{R}$ indices $[\mathrm{I}>2 \sigma(\mathrm{I})]$ & $\mathrm{R} 1=0.0423, \mathrm{wR} 2=0.1182$ \\
\hline $\mathrm{R}$ indices (all data) & $\mathrm{R} 1=0.0483, \mathrm{wR} 2=0.1227$ \\
\hline Absolute structure parameter & $0.042(12)$ \\
\hline Largest diff. peak and hole & 0.918 and -0.601 e. $\AA^{-3}$ \\
\hline
\end{tabular}


Table S2. Selected Bond lengths $[\AA]$ and angles $\left[^{\circ}\right]$ for $1 \cdot 6 \mathrm{THF}$.

\begin{tabular}{|c|c|}
\hline $\mathrm{Zn}(1)-\mathrm{O}(2)$ & $1.955(3)$ \\
\hline $\mathrm{Zn}(1)-\mathrm{O}(1)$ & $1.983(3)$ \\
\hline $\mathrm{Zn}(1)-\mathrm{N}(3)$ & $2.080(4)$ \\
\hline $\mathrm{Zn}(1)-\mathrm{N}(2)$ & $2.080(4)$ \\
\hline $\mathrm{Zn}(1)-\mathrm{N}(5) \# 1$ & $2.081(4)$ \\
\hline $\mathrm{Zn}(2)-\mathrm{O}(3)$ & $1.976(3)$ \\
\hline $\mathrm{Zn}(2)-\mathrm{O}(4)$ & $1.982(3)$ \\
\hline $\mathrm{Zn}(2)-\mathrm{N}(6)$ & $2.073(4)$ \\
\hline $\mathrm{Zn}(2)-\mathrm{N}(1)$ & $2.092(4)$ \\
\hline $\mathrm{Zn}(2)-\mathrm{N}(7)$ & $2.093(4)$ \\
\hline $\mathrm{O}(2)-\mathrm{Zn}(1)-\mathrm{O}(1)$ & $94.63(13)$ \\
\hline $\mathrm{O}(2)-\mathrm{Zn}(1)-\mathrm{N}(3)$ & $146.93(14)$ \\
\hline $\mathrm{O}(1)-\mathrm{Zn}(1)-\mathrm{N}(3)$ & $89.27(14)$ \\
\hline $\mathrm{O}(2)-\mathrm{Zn}(1)-\mathrm{N}(2)$ & $89.56(14)$ \\
\hline $\mathrm{O}(1)-\mathrm{Zn}(1)-\mathrm{N}(2)$ & $164.18(14)$ \\
\hline $\mathrm{N}(3)-\mathrm{Zn}(1)-\mathrm{N}(2)$ & $79.07(14)$ \\
\hline $\mathrm{O}(2)-\mathrm{Zn}(1)-\mathrm{N}(5) \# 1$ & $105.12(16)$ \\
\hline $\mathrm{O}(1)-\mathrm{Zn}(1)-\mathrm{N}(5) \# 1$ & $93.61(15)$ \\
\hline $\mathrm{N}(3)-\mathrm{Zn}(1)-\mathrm{N}(5) \# 1$ & $107.39(15)$ \\
\hline $\mathrm{N}(2)-\mathrm{Zn}(1)-\mathrm{N}(5) \# 1$ & $100.02(15)$ \\
\hline $\mathrm{O}(3)-\mathrm{Zn}(2)-\mathrm{O}(4)$ & $93.70(13)$ \\
\hline $\mathrm{O}(3)-\mathrm{Zn}(2)-\mathrm{N}(6)$ & $150.23(14)$ \\
\hline $\mathrm{O}(4)-\mathrm{Zn}(2)-\mathrm{N}(6)$ & $89.13(14)$ \\
\hline $\mathrm{O}(3)-\mathrm{Zn}(2)-\mathrm{N}(1)$ & $97.42(15)$ \\
\hline $\mathrm{O}(4)-\mathrm{Zn}(2)-\mathrm{N}(1)$ & $101.16(15)$ \\
\hline $\mathrm{N}(6)-\mathrm{Zn}(2)-\mathrm{N}(1)$ & $111.09(15)$ \\
\hline $\mathrm{O}(3)-\mathrm{Zn}(2)-\mathrm{N}(7)$ & $88.23(13)$ \\
\hline $\mathrm{O}(4)-\mathrm{Zn}(2)-\mathrm{N}(7)$ & $159.05(14)$ \\
\hline $\mathrm{N}(6)-\mathrm{Zn}(2)-\mathrm{N}(7)$ & $79.18(15)$ \\
\hline $\mathrm{N}(1)-\mathrm{Zn}(2)-\mathrm{N}(7)$ & $99.26(16)$ \\
\hline
\end{tabular}

Symmetry transformations used to generate equivalent atoms:

\#1 -y,x-y-1,z \#2-x+y+1,-x,z 
Table S3. Crystal data and structure refinement for 1·6Pentanol

\begin{tabular}{|c|c|}
\hline Identification code & 1.6Pentanol \\
\hline Empirical formula & $\mathrm{C}_{258} \mathrm{H}_{324} \mathrm{~N}_{24} \mathrm{O}_{18} \mathrm{Zn}_{6}$ \\
\hline Formula weight & 4441.63 \\
\hline Temperature & $273(2) \mathrm{K}$ \\
\hline Wavelength & $0.71073 \AA$ \\
\hline Crystal system & Trigonal \\
\hline Space group & $\mathrm{R} 3$ \\
\hline \multicolumn{2}{|l|}{ Unit cell dimensions } \\
\hline & $\mathrm{a}=45.0370(19) \AA \quad$ alpha $=90^{\circ}$ \\
\hline & $\mathrm{b}=45.0370(19) \AA \quad$ beta $=90^{\circ}$ \\
\hline & $\mathrm{c}=10.3862(9) \AA \quad$ gamma $=120^{\circ}$ \\
\hline Volume, Z & $18244.3(19) \AA^{3}, 3$ \\
\hline Density (calculated) & $1.213 \mathrm{Mg} / \mathrm{m}^{3}$ \\
\hline Absorption coefficient & $0.648 \mathrm{~mm}^{-1}$ \\
\hline $\mathrm{F}(000)$ & 7092 \\
\hline Crystal size & $0.15 \times 0.06 \times 0.06 \mathrm{~mm}$ \\
\hline Theta range for data collection & 2.22 to $25.00^{\circ}$ \\
\hline Limiting indices & $-53<\mathrm{h}<52,-53<\mathrm{k}<48,-12<1<12$ \\
\hline Reflections collected & 31800 \\
\hline Independent reflections & $14160($ Rint $=0.0725)$ \\
\hline Completeness to theta & $25.00^{\circ} / 99.8 \%$ \\
\hline Refinement method & Full-matrix least-squares on $\mathrm{F}^{2}$ \\
\hline Data / restraints / parameters & $14160 / 16 / 839$ \\
\hline Goodness-of-fit on $\mathrm{F}^{\wedge} 2$ & 1.001 \\
\hline Final $R$ indices $[\mathrm{I}>2 \sigma(\mathrm{I})]$ & $\mathrm{R} 1=0.0646, \mathrm{wR} 2=0.1462$ \\
\hline $\mathrm{R}$ indices (all data) & $\mathrm{R} 1=0.1262, \mathrm{wR} 2=0.1802$ \\
\hline Absolute structure parameter & $0.06(2)$ \\
\hline Largest diff. peak and hole & 0.571 and -0.321 e. $\AA^{-3}$ \\
\hline
\end{tabular}


Table S4. Bond lengths $[\AA]$ and angles $\left[{ }^{\circ}\right]$ for 1.6Pentanol.

\begin{tabular}{lr}
\hline $\mathrm{Zn}(1)-\mathrm{O}(1)$ & $1.955(7)$ \\
$\mathrm{Zn}(1)-\mathrm{O}(2)$ & $1.980(7)$ \\
$\mathrm{Zn}(1)-\mathrm{N}(2)$ & $2.095(8)$ \\
$\mathrm{Zn}(1)-\mathrm{N}(5) \# 1$ & $2.101(9)$ \\
$\mathrm{Zn}(1)-\mathrm{N}(3)$ & $2.102(9)$ \\
$\mathrm{Zn}(2)-\mathrm{O}(4)$ & $1.976(8)$ \\
$\mathrm{Zn}(2)-\mathrm{O}(3)$ & $1.980(7)$ \\
$\mathrm{Zn}(2)-\mathrm{N}(7)$ & $2.072(9)$ \\
$\mathrm{Zn}(2)-\mathrm{N}(6)$ & $2.073(8)$ \\
$\mathrm{Zn}(2)-\mathrm{N}(1)$ & $2.101(9)$ \\
& \\
$\mathrm{O}(1)-\mathrm{Zn}(1)-\mathrm{O}(2)$ & $94.7(3)$ \\
$\mathrm{O}(1)-\mathrm{Zn}(1)-\mathrm{N}(2)$ & $88.3(3)$ \\
$\mathrm{O}(2)-\mathrm{Zn}(1)-\mathrm{N}(2)$ & $150.3(3)$ \\
$\mathrm{O}(1)-\mathrm{Zn}(1)-\mathrm{N}(5) \# 1$ & $100.9(3)$ \\
$\mathrm{O}(2)-\mathrm{Zn}(1)-\mathrm{N}(5) \# 1$ & $97.0(3)$ \\
$\mathrm{N}(2)-\mathrm{Zn}(1)-\mathrm{N}(5) \# 1$ & $111.4(3)$ \\
$\mathrm{O}(1)-\mathrm{Zn}(1)-\mathrm{N}(3)$ & $159.4(3)$ \\
$\mathrm{O}(2)-\mathrm{Zn}(1)-\mathrm{N}(3)$ & $87.6(3)$ \\
$\mathrm{N}(2)-\mathrm{Zn}(1)-\mathrm{N}(3)$ & $79.9(3)$ \\
$\mathrm{N}(5) \# 1-\mathrm{Zn}(1)-\mathrm{N}(3)$ & $99.0(3)$ \\
$\mathrm{O}(4)-\mathrm{Zn}(2)-\mathrm{O}(3)$ & $95.0(3)$ \\
$\mathrm{O}(4)-\mathrm{Zn}(2)-\mathrm{N}(7)$ & $146.6(3)$ \\
$\mathrm{O}(3)-\mathrm{Zn}(2)-\mathrm{N}(7)$ & $88.9(3)$ \\
$\mathrm{O}(4)-\mathrm{Zn}(2)-\mathrm{N}(6)$ & $89.8(3)$ \\
$\mathrm{O}(3)-\mathrm{Zn}(2)-\mathrm{N}(6)$ & $162.4(3)$ \\
$\mathrm{N}(7)-\mathrm{Zn}(2)-\mathrm{N}(6)$ & $78.0(3)$ \\
$\mathrm{O}(4)-\mathrm{Zn}(2)-\mathrm{N}(1)$ & $104.5(4)$ \\
$\mathrm{O}(3)-\mathrm{Zn}(2)-\mathrm{N}(1)$ & $94.6(4)$ \\
$\mathrm{N}(7)-\mathrm{Zn}(2)-\mathrm{N}(1)$ & $108.2(3)$ \\
$\mathrm{N}(6)-\mathrm{Zn}(2)-\mathrm{N}(1)$ & $100.6(3)$ \\
& \\
\hline
\end{tabular}

Symmetry transformations used to generate equivalent atoms:

$\# 1-y+1, x-y+1, z \quad \# 2-x+y,-x+1, z$ 
Figure S1 The molecular structure of $1 \cdot 6 \mathrm{THF}$.
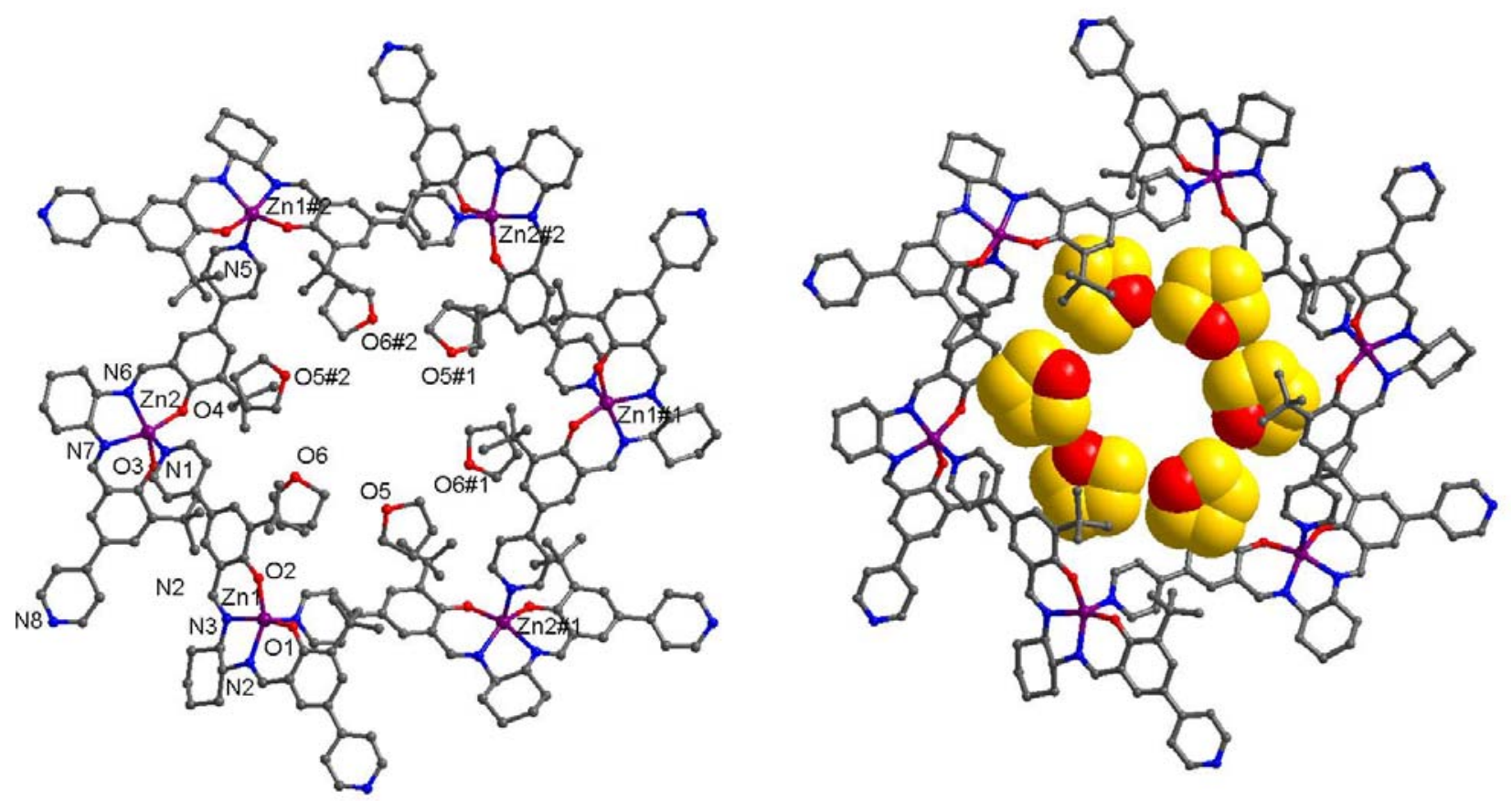

Figure S2 The molecular structure of $\mathbf{1} \cdot 6$ pentanol
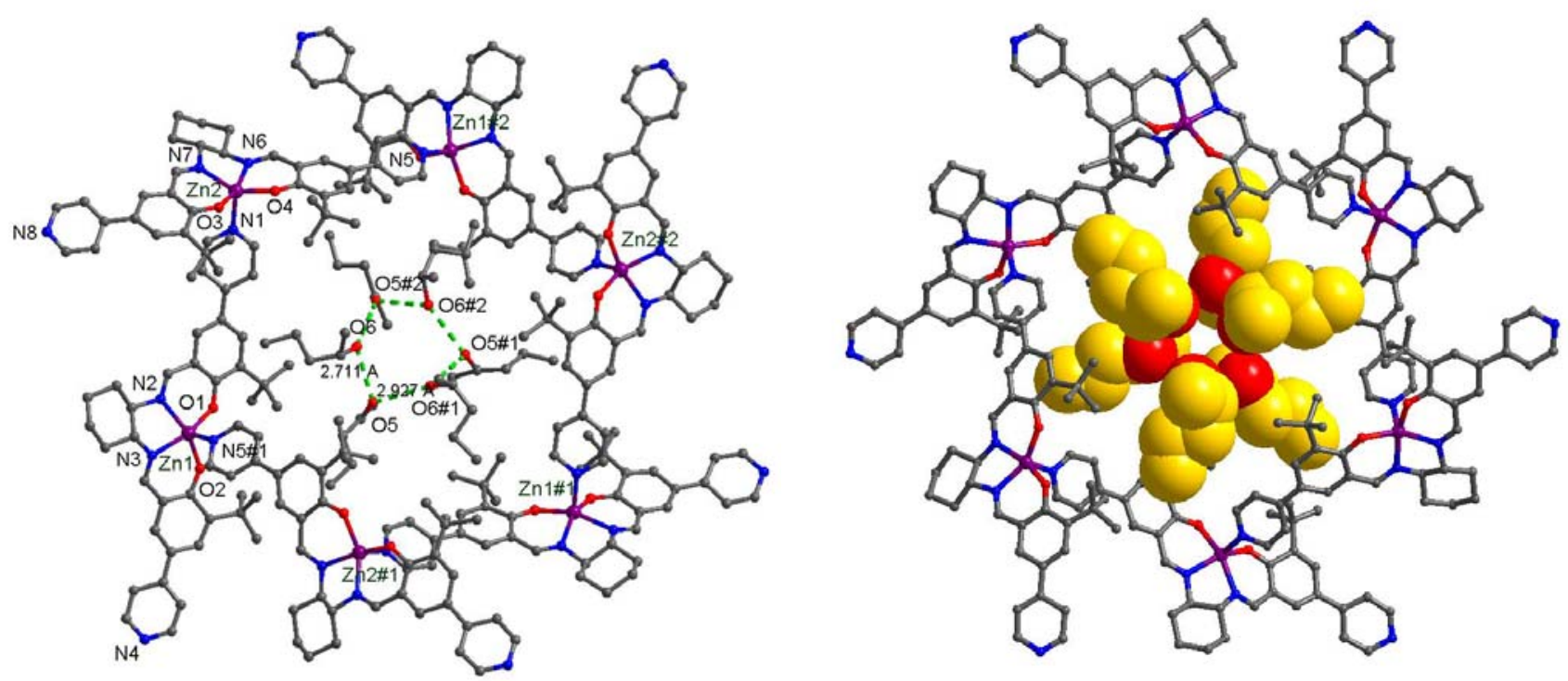
Figure S3 $\mathrm{CH} \cdots \pi$ interactions between the butyl groups and the coordinated pyridine rings of $\mathbf{L}$ direct $\mathrm{Zn}_{6} \mathbf{L}_{6}$ macrocycles assembling into nanotubes along the crystallographic c-axis.
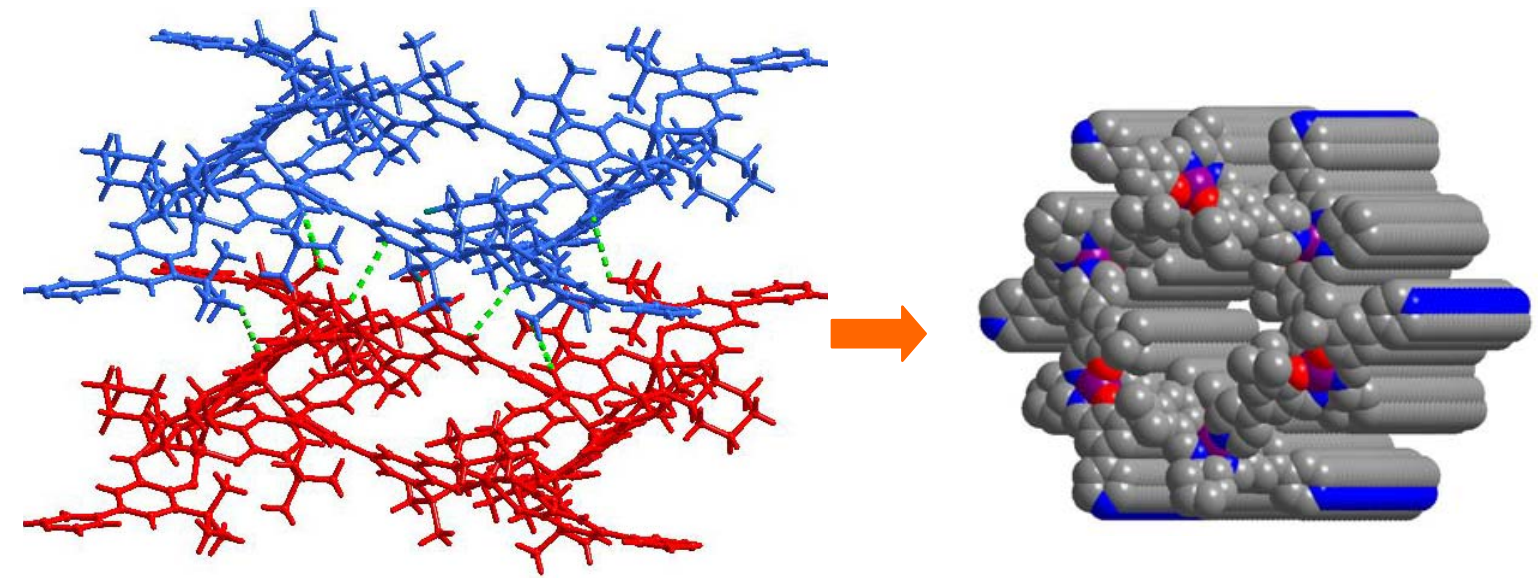

$\mathrm{d}=3.0$ to $3.2 \mathrm{~A}$ for $1.6 \mathrm{THF}$ and 1.6 Pentanol

Figure S4 Intermolecular $\pi-\pi$ interaction between $\mathrm{Zn}_{6} \mathbf{L}_{6}$ macrocycles in the two compounds

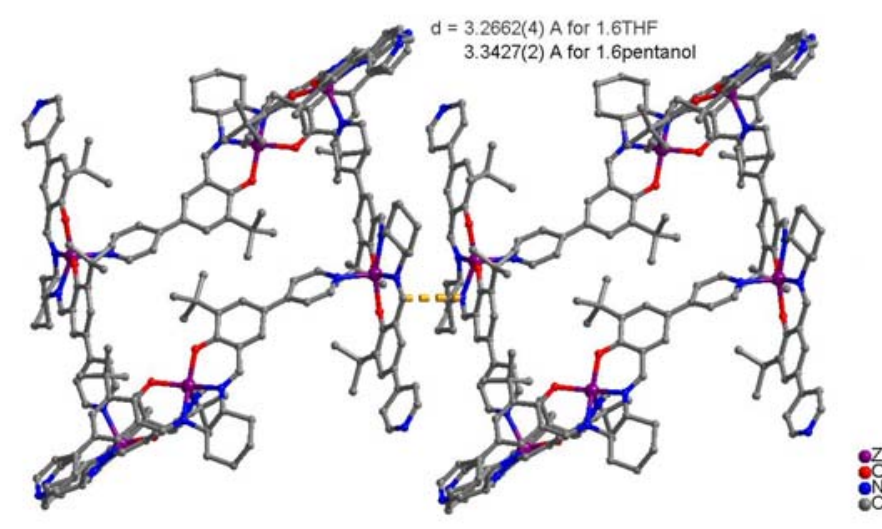

Figure S5. $\mathrm{CH} \cdots \pi$ interactions between uncoordinated pyridyl groups and aromatic rings of $\mathrm{Zn}_{6} \mathbf{L}_{6}$ macrocycles in the two compounds.

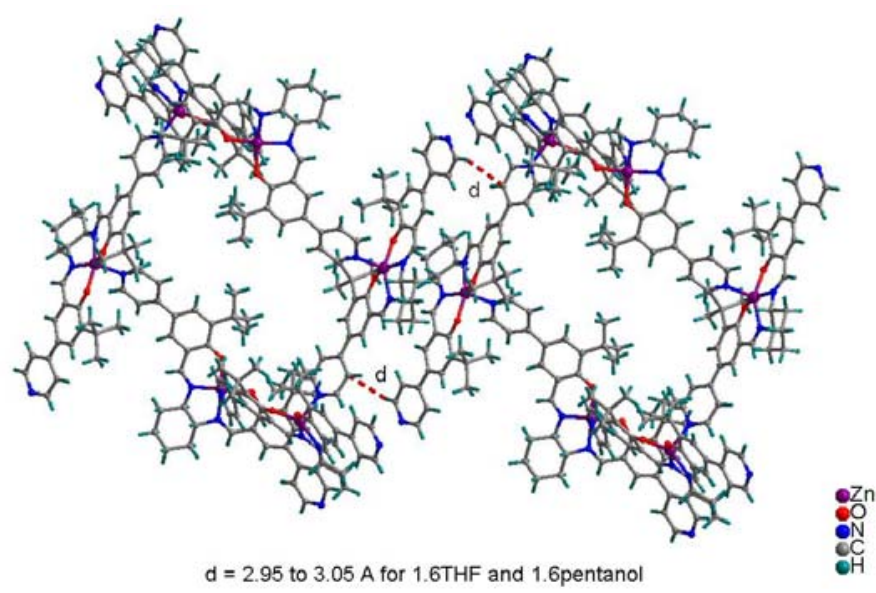


Figure S6. $\mathrm{CH} \cdots \mathrm{N}$ interactions between uncoordinated pyridyl groups and aromatic rings of $\mathrm{Zn}_{6} \mathbf{L}_{6}$ macrocycles in the two compounds.

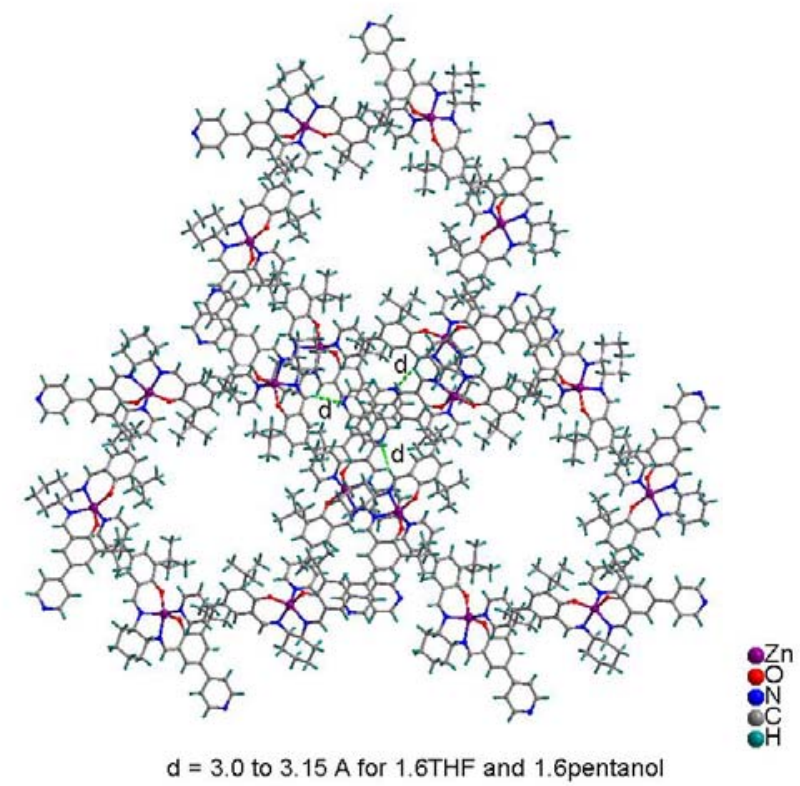

Figure S7. A view of the packing of $\mathrm{Zn}_{6} \mathbf{L}_{6}$ cages along the c-axis (Top) and its space-filling mode (Bottom) of the four compounds.

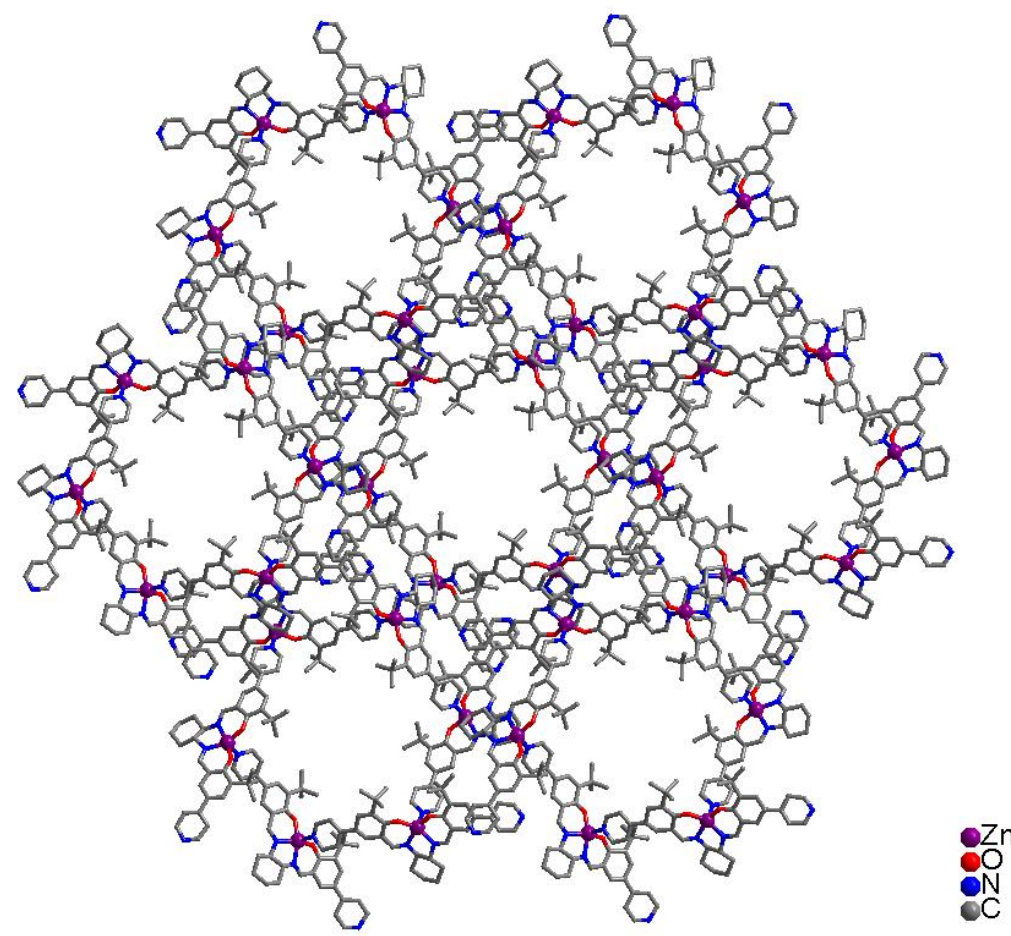


Figure S8. The PXRD patterns of 1·6THF, 1, 1·6(2-penatnol), 1·6(2-butanol), 1·6(3-methyl-2-butanol) and the simulated PXRD pattern of $1 \cdot 6 \mathrm{THF}$.

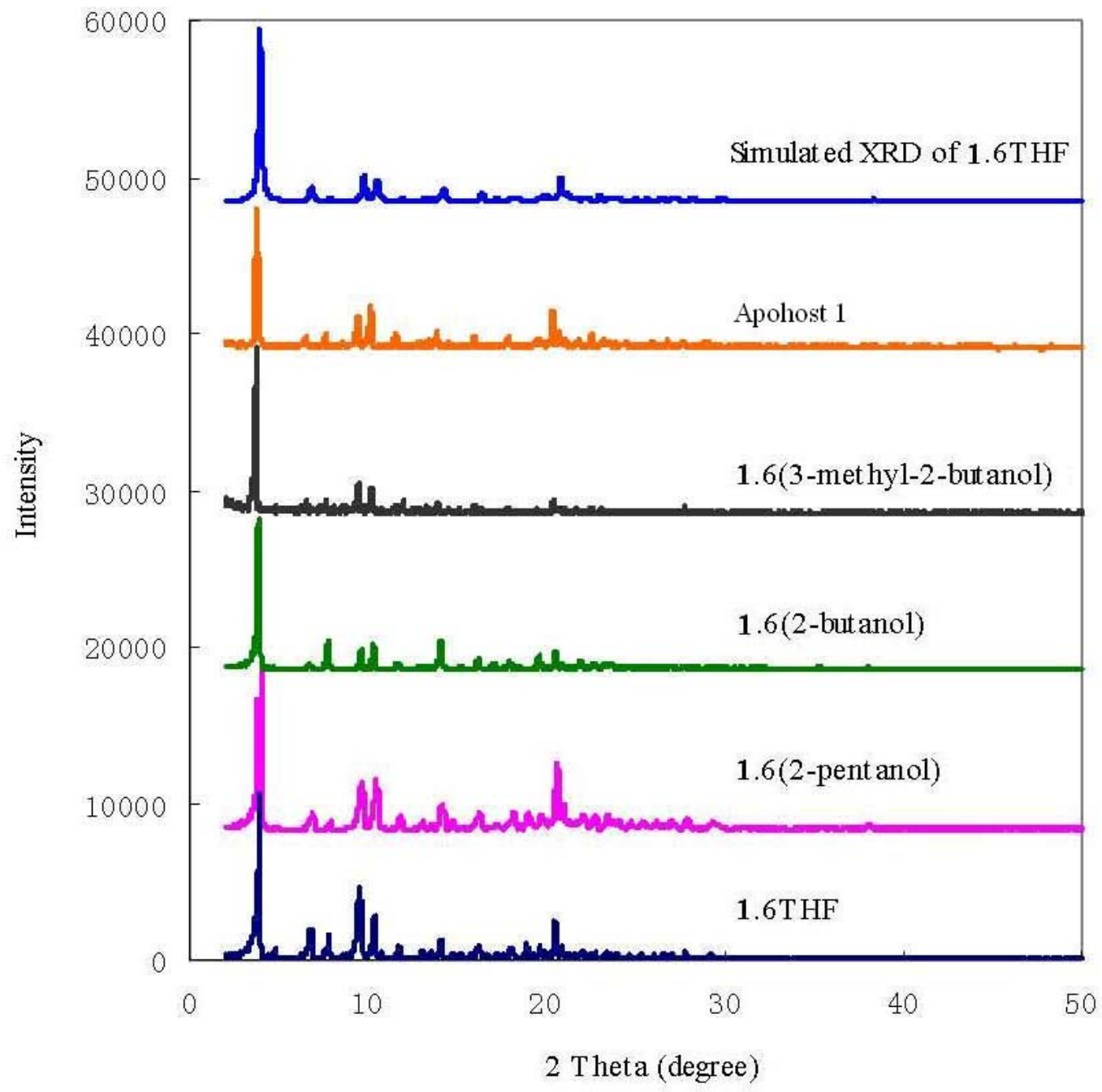


Figure S9. The PXRD patterns of 1 after the $3^{\text {rd }}$ and $5^{\text {th }}$ cycles of separation of 2-butanol

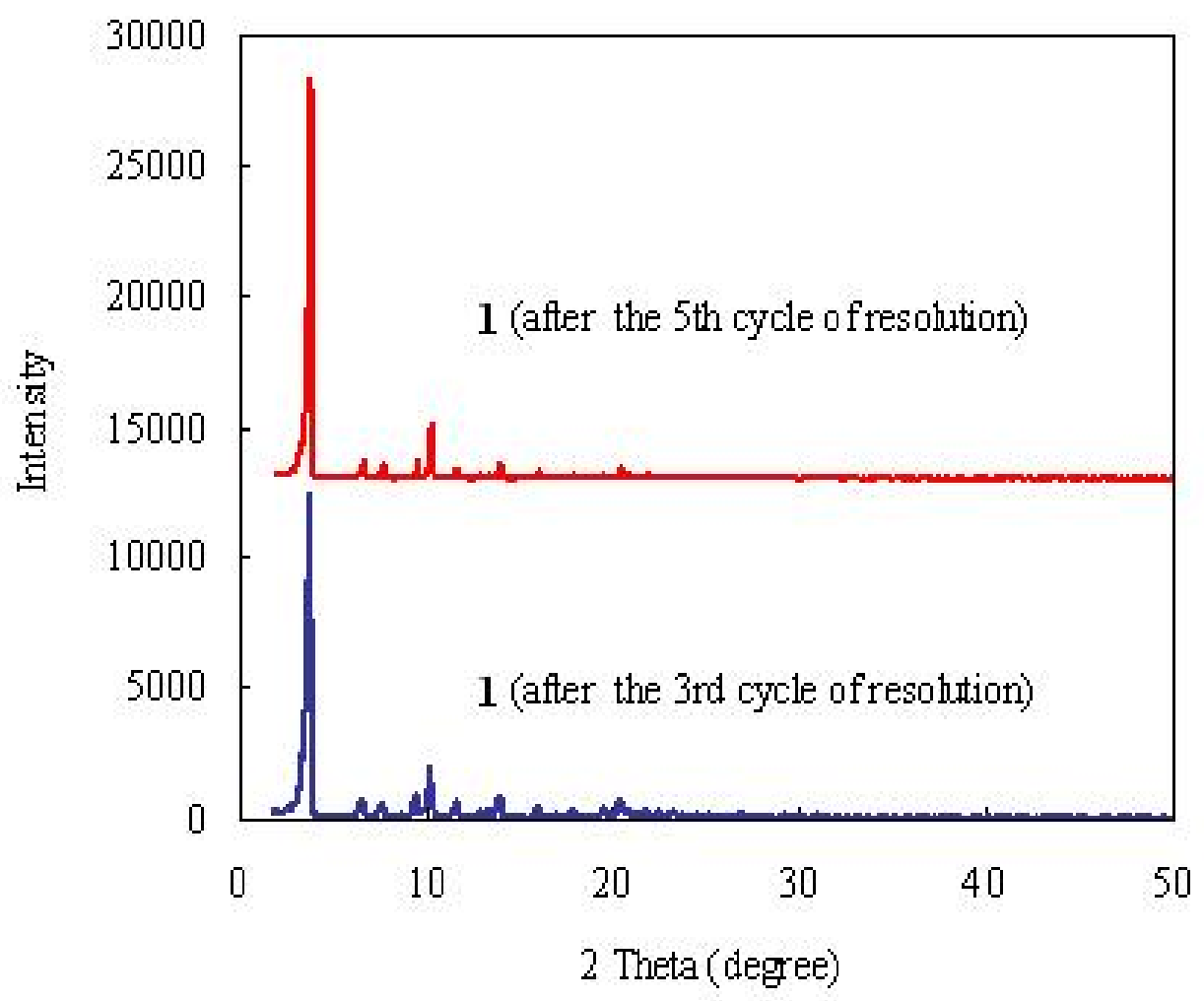

Figure S10. TGA curves of 1.6Guests. (9.96\% for 1.6THF, $10.2 \%$ for $1.6(2$-butanol) and $11.9 \%$ for 1.6(2-pentanol) and 1.6(3-methyl-2-butanol)).

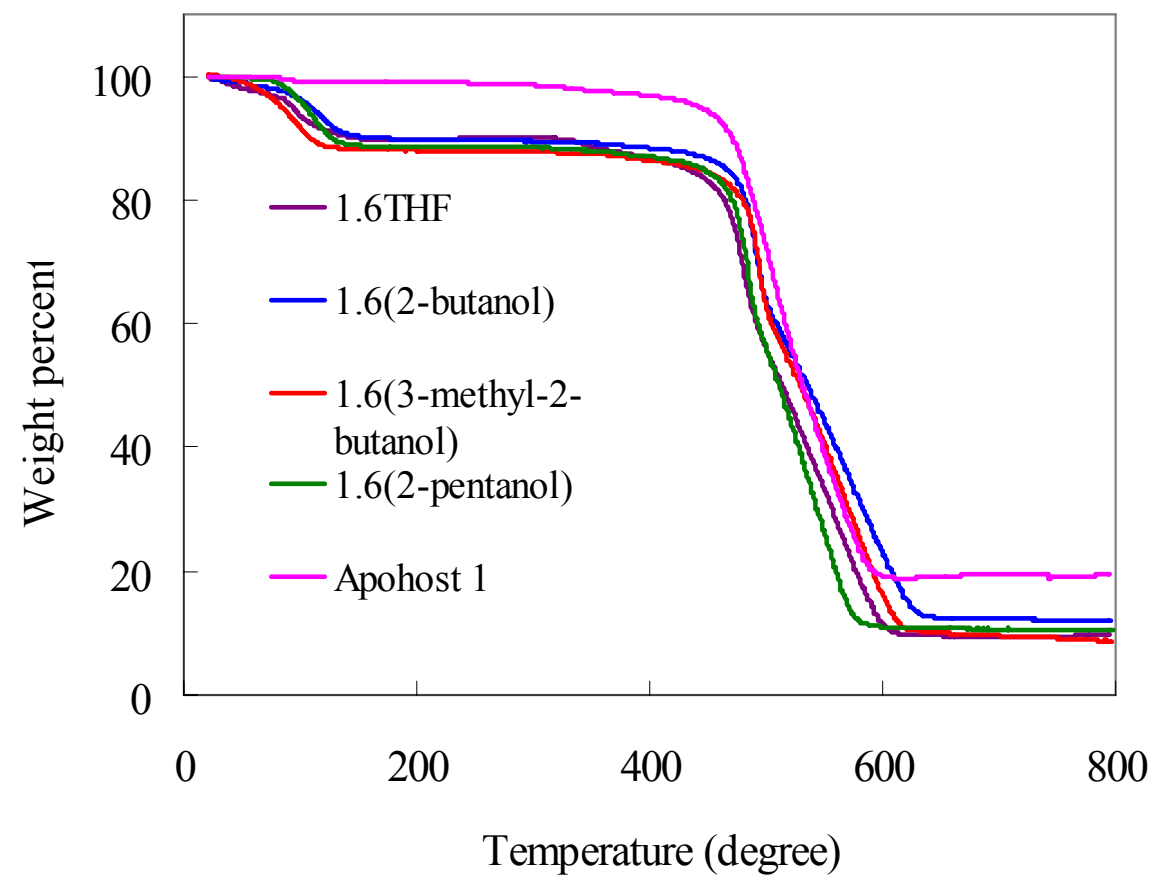


Figure S11. $\mathrm{N}_{2}$ adsorption isotherm of $\mathbf{1}$.

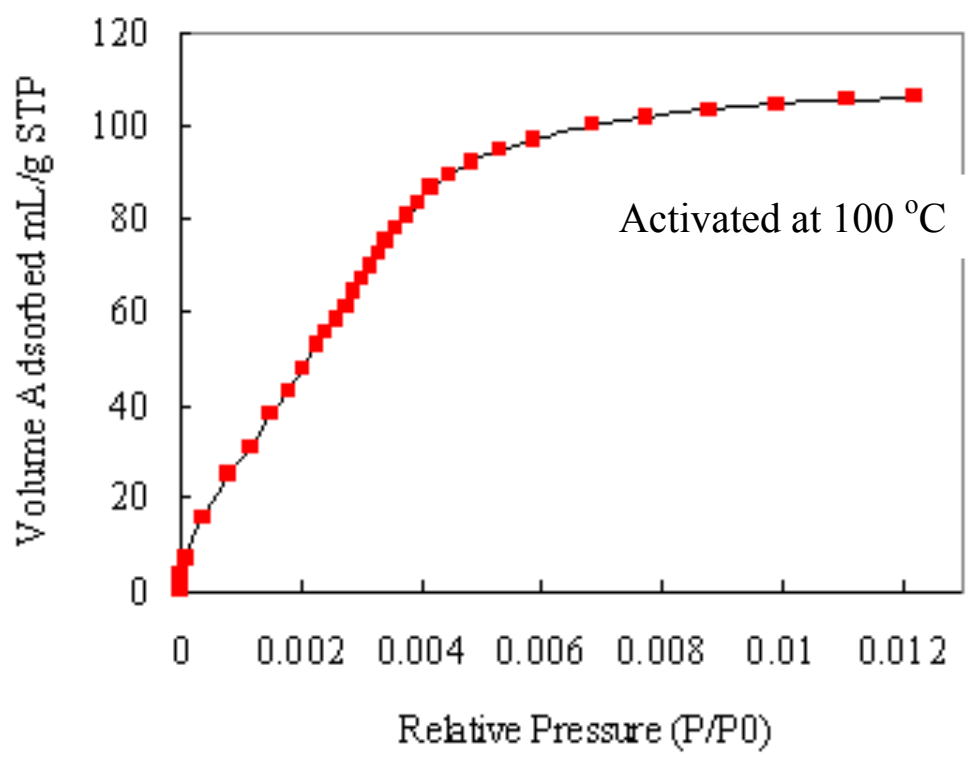

Figure S12. (a) Chiral GC results of the desorbed (R)-3-methyl-2-Butanol from (R)-1 and racemic 3-methyl-2-Butanol (black line). (b) Chiral GC results of the desorbed (R)-2-pentanol from (R)-1 and racemic 2-pentanol (black line).

(a)
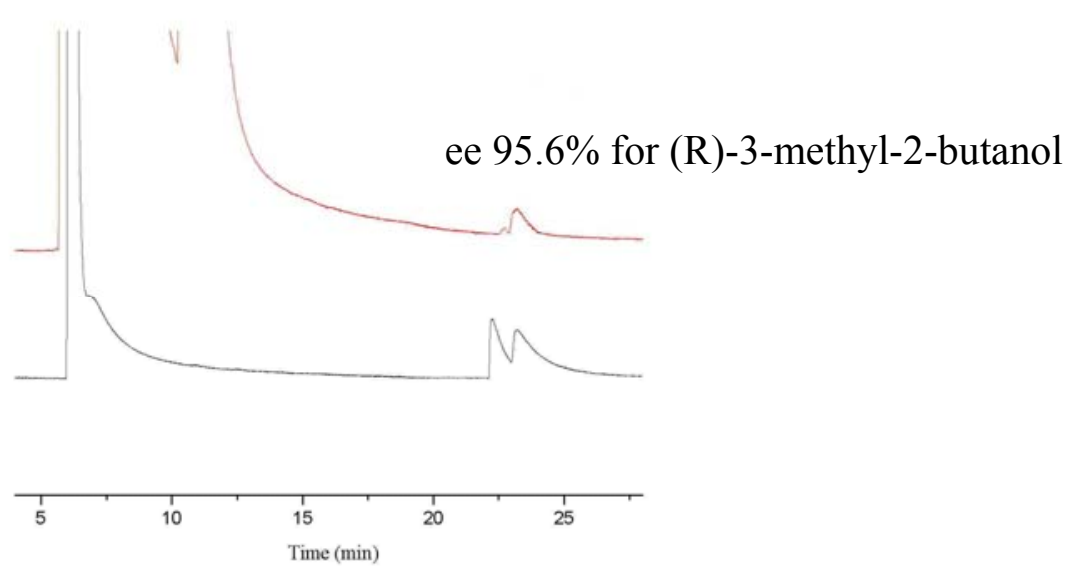

(b)

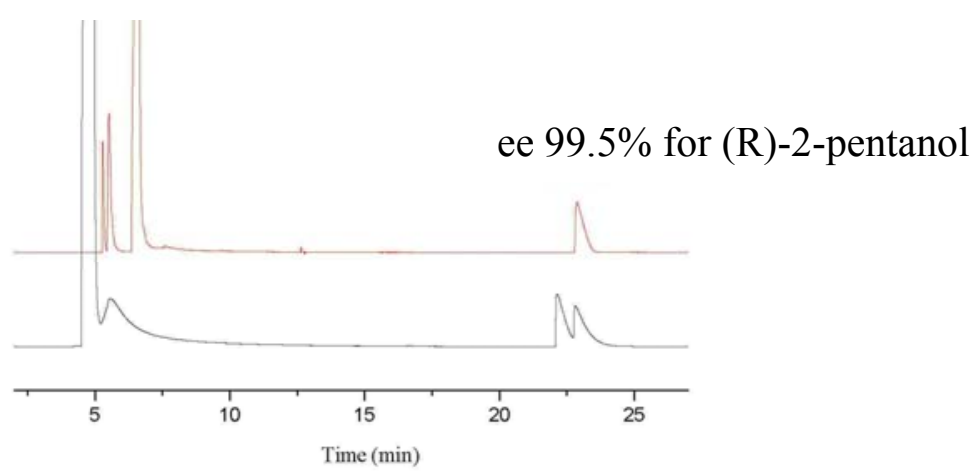


Figure S13. Chiral GC results of recycling and reuse of (R)-1 for separation of racemic 2-butanol (a-b: the first cycle and the fifth cycle, respectively. red line: desorbed sample; black line: racemic sample).

(a)

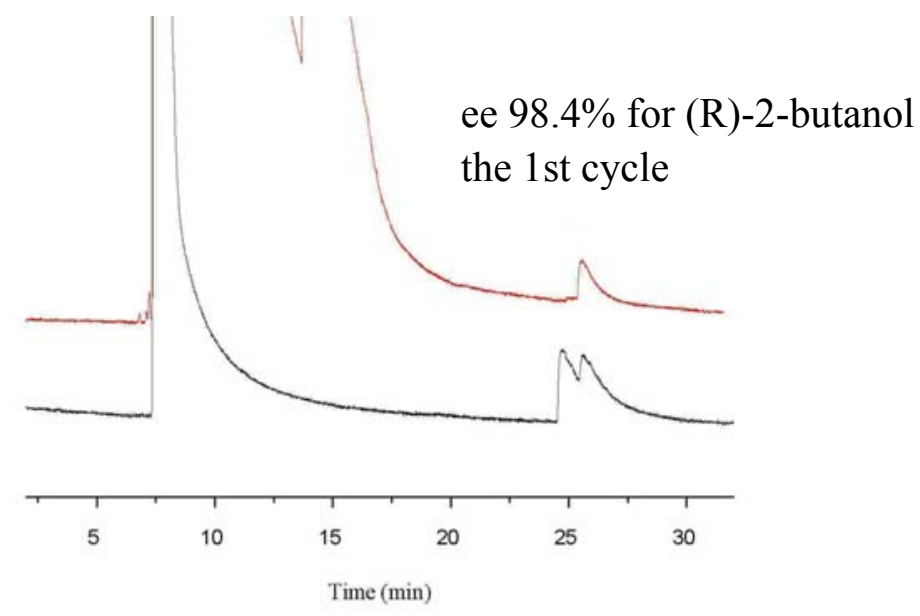

(b)

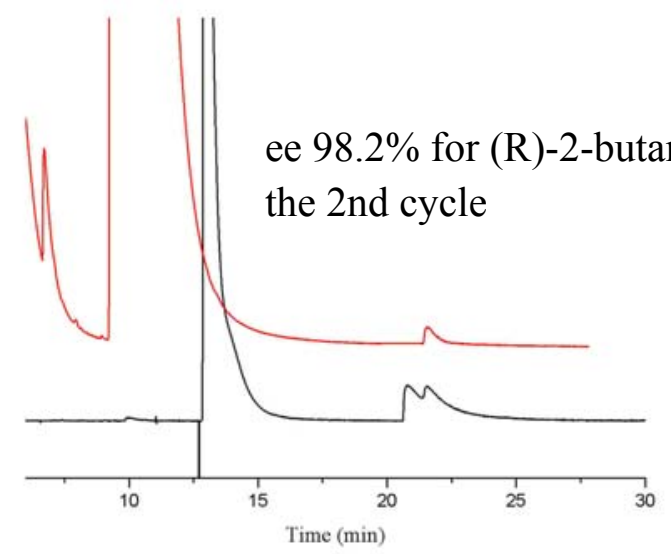

(d)

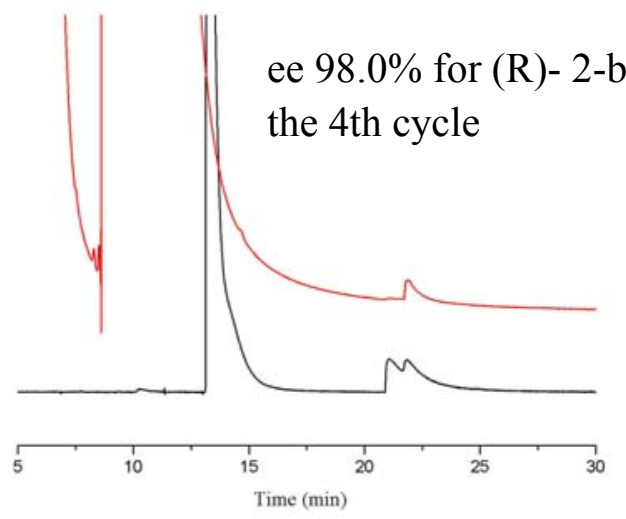

(c)

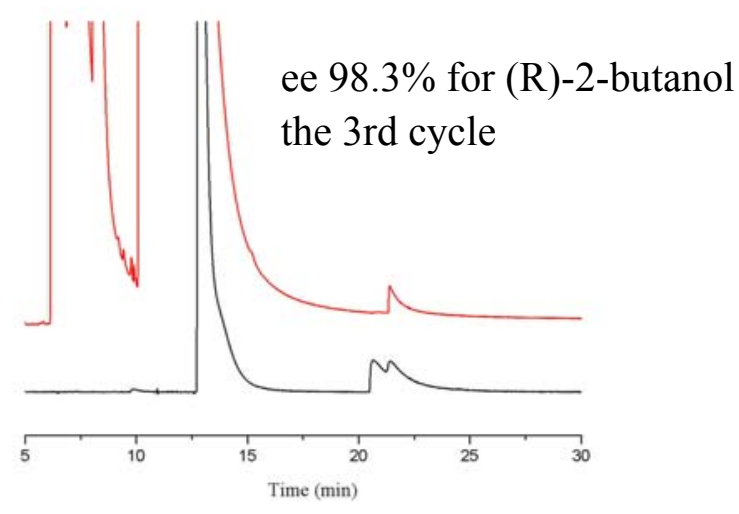

(e)

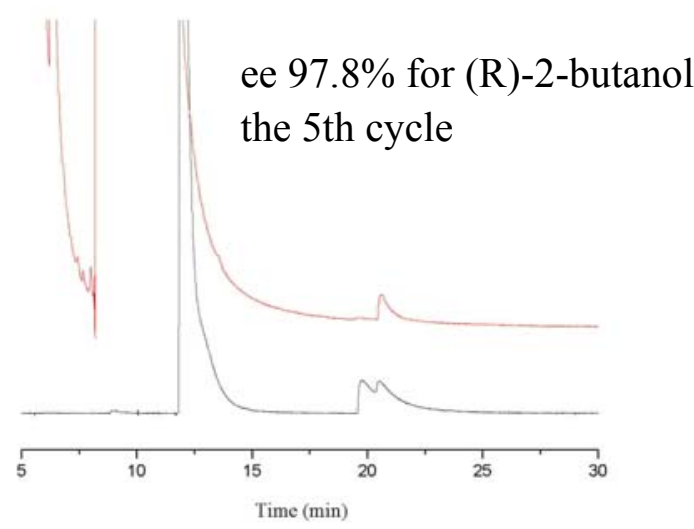


Figure S14. (a) Chiral GC results of the desorbed (S)-2-butanol from (S)-1 and racemic 2-pentanol (black line). (b) Chiral GC results of the desorbed (S)-3-methyl-2-Butanol from (S)-1 and racemic 3-methyl-2-Butanol (black line). (c) Chiral GC results of the desorbed (S)-2-pentanol from (S)-1 and racemic 2-pentanol (black line).

(a)

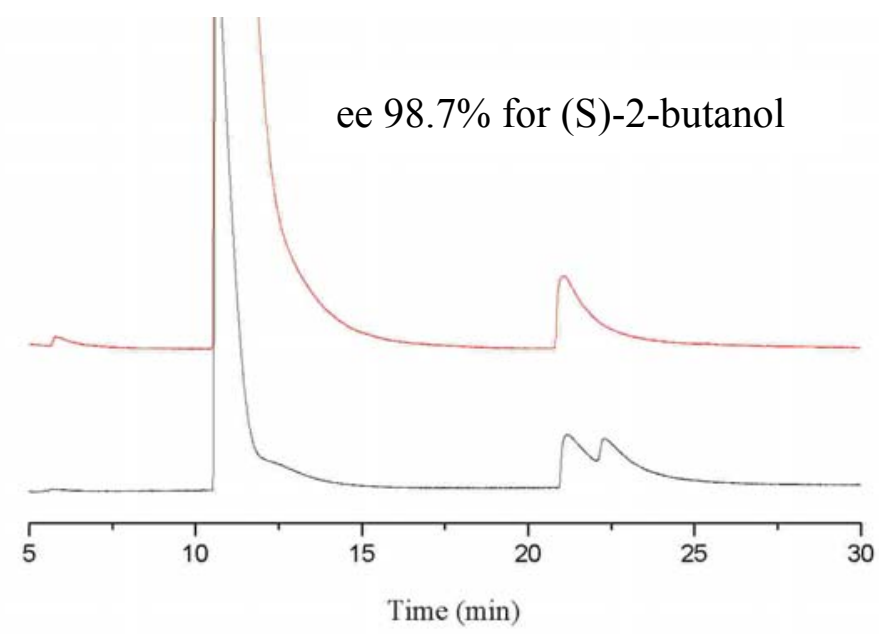

(b)

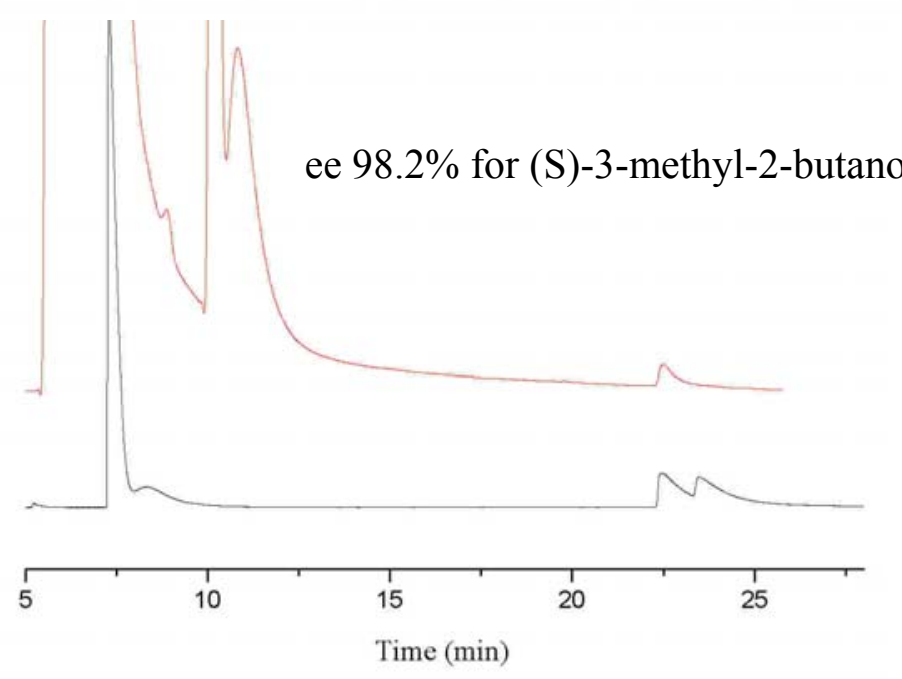

(c)

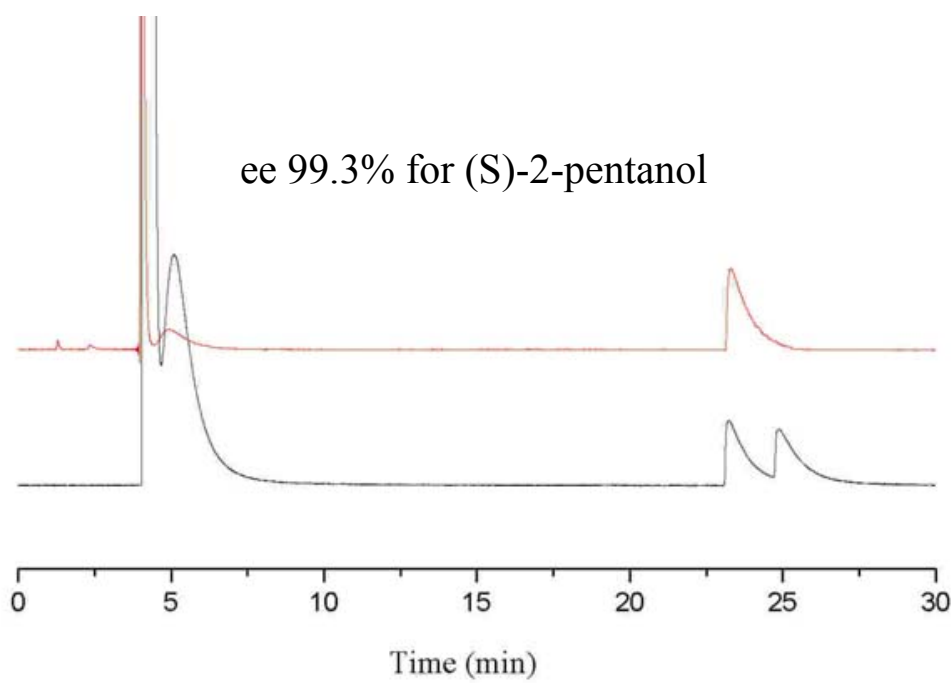


Figure S15. CD spectra of $\mathbf{1}$. The opposite handedness of (R)- and (S)-1 could been seen clearly.

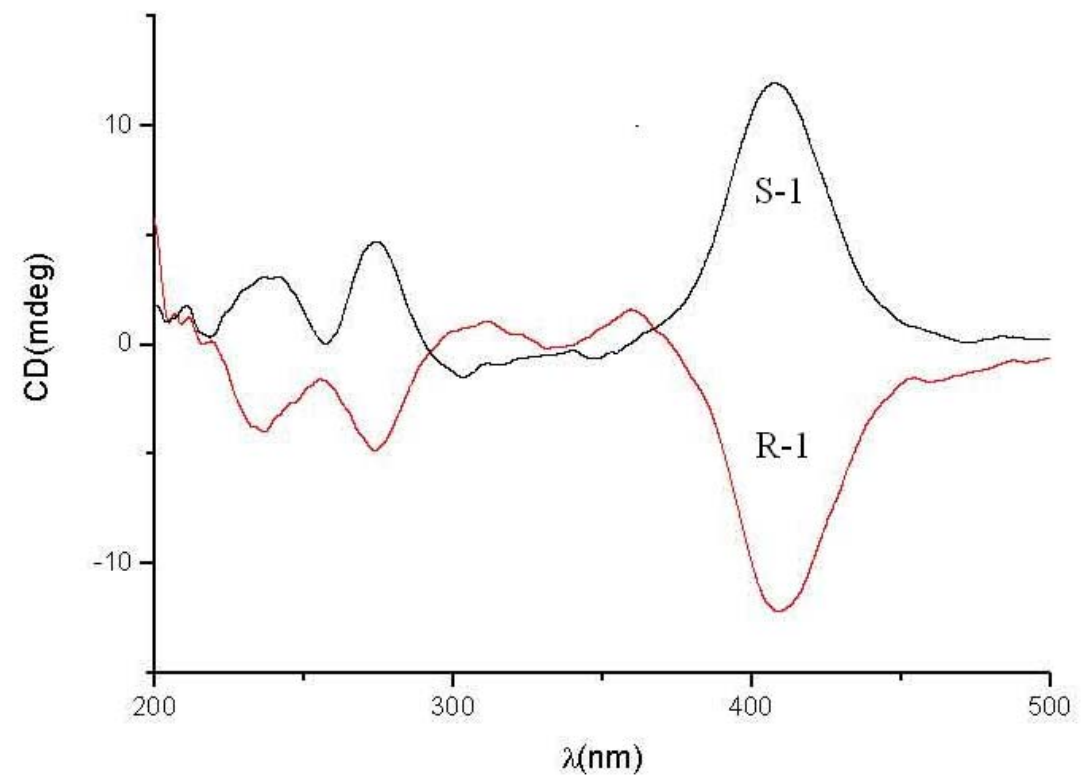

